\title{
Tipo de arcada y plano terminal molar de la dentición temporal y su correlación con las clases de maloclusión de la dentición permanente
}

\author{
Terminal plane and dental arch form in temporal dentition correlated \\ to angle class malocclusion in permanent dentition
}

\author{
Ana Cecilia Pascual Serna \\ Universidad Nacional Daniel Alcides Carrión \\ ceciliapascuals@hotmail.com
}

\author{
Eduardo López Pagán² \\ Universidad Nacional Daniel Alcides Carrión \\ eduardolopezpagan@yahoo.es
}

\section{RESUMEN}

El objetivo fue establecer la correlación del tipo de arcada y plano terminal molar de la dentición temporal con las clases de maloclusión de Angle en niños de 5 años de edad que acuden al Hospital Nacional "Daniel Alcides Carrión"de Cerro de Pasco, 2010 - 2011. La investigación se desarrolló bajo un diseño descriptivo longitudinal prospectivo. La muestra estuvo conformada por 40 niños escogidos por muestreo no probabilístico. Se realizó inicialmente la evaluación clínica del tipo de arcada y plano terminal molar de la dentición temporal y posteriormente al erupcionar las primeras molares permanentes se verificó la correlación con las clases de maloclusión de Angle, el tratamiento estadístico de la hipótesis fue con la Chi Cuadrada. Entre los resultados podemos mencionar que existe diferencia estadística significativa para asumir que el tipo de plano terminal molar y tipo de arcada de la dentición temporal se correlaciona con las clases de maloclusión de Angle, teniendo en consideración que la $X 2 c=12,724>\times 2 \dagger=9,488(4 \mathrm{gl}-95 \%)$, rechazándose la Ho. En conclusión, el tipo de plano terminal molar recto y el tipo de arcada abierta de la dentición temporal se relaciona con mayor frecuencia con la normoclusión y la Clase I de maloclusión. Mientras que el tipo de plano terminal molar escalón mesial y el tipo de arcada cerrada de la dentición temporal se relaciona con la Clase III de maloclusión.

Palabras clave: Tipo de arcada, plano terminal molar, maloclusión de Angle, dentición temporal, dentición permanente.

\section{ABSTRACT}

The study objective was to establish the correlation between dental arch form and molar terminal plane in temporal dentition with angle class malocclusion in 5-year-old children who attended in the Hospital Nacional "Daniel Alcides Carrión" at Cerro de Pasco, 2010 - 2011. The research used a prospective, longitudinal descriptive design. The sample consisted of 40 children chosen by a nonprobability sampling. For a start, a clinical evaluation of the dental arch form and molar terminal plane in temporal dentition was performed. Then, after the first permanent molars erupted, a correlation with malocclusion angle classes was observed; the statistical treatment hypothesis was done with Chi Square. Results indicated a significant statistical difference to assume that molar terminal plane and dental arch form in temporal dentition is correlated with malocclusion angle classes, considering 12,724 $\mathrm{X} 2 \mathrm{c}=>\mathrm{X} 2 \mathrm{~T}=9,488$ (4GL-95\%), rejecting the $\mathrm{Ho}$. Concluding, the straight terminal plane and open dental arch form in temporal dentition is associated with increased frequency of normoclusion and Class I malocclusion. While the mesial step terminal plane type and closed dental arch form in temporal dentition is related to Class III malocclusion.

Keywords: Arch form, type of molar terminal plane, Angle malocclusion, temporal and permanent dentition.

Historial del artículo:

Recibido: 21 de octubre de 2015. Aprobado: 9 de diciembre de 2015. Disponible en línea: 30 de diciembre de 2015

1 Magister en Odontología, docente de Ciencias Básicas de la Universidad Nacional Daniel Alcides Carrión.

2 Especialista en Periodoncia, Magister en Salud Pública, docente de Periodoncia de la Universidad Nacional Daniel Alcides Carrión. 


\section{INTRODUCCIÓN}

La maloclusión se considera como una de las enfermedades más prevalentes de la cavidad oral en nuestro país, por ello resulta importante reconocer características de la dentición temporal que puedan influenciar en la maloclusión de la dentición permanente.

La primera dentición del ser humano es la temporal o decidua la cual comprende una serie de características, dichas características nos deben ayudar a establecer un patrón predictivo, probablemente el resultado sea una dentición permanente aceptable con dientes alineados y relaciones oclusales que permitirán una correcta función y estética.

En la presente investigación se observó fundamentalmente los tipos de arcadas y los planos terminales de la dentición decidua y si estas características nos podrían ayudar a predecir maloclusión en la dentición permanente.

En la actualidad se busca diagnosticar maloclusiones tempranamente con el fin de interceptar una posible maloclusión y plantear un plan de tratamiento inmediato y oportuno, razón por la cual se ha considerado importante revisar este tema.

El problema de investigación fue ¿̇Cuál es la correlación del tipo de plano terminal molar y tipo de arcada en la dentición temporal con las clases de maloclusión de Angle en niños de 5 años que acuden al Hospital Nacional "Daniel Alcides Carrión" de la ciudad de Cerro de Pasco, 2010 - 2011?. El objetivo de la investigación fue establecer la correlación entre el tipo de plano terminal molar y tipo de arcada de la dentición temporal con las clases de maloclusión de Angle.

La investigación se justifica en la importancia de diagnosticar oportunamente algunas alteraciones en la oclusión decidua que futuramente traerían consecuencias desfavorables en la dentición permanente y por consiguiente en la salud en general.

La metodología utilizada estuvo basada en la evaluación clínica inicial realizada en los niños de cinco años que presentaban en su totalidad dentición decidua característica odontológica propia de su edad. Donde se diagnosticó el tipo de plano Terminal molar y tipo de arcada de la dentición temporal. La evaluación clínica final se hizo después del primer año para evaluar la posición de las primeras molares permanentes que son los dientes claves o llaves para la adecuada alineación de los demás dientes permanentes. Se determinó la evaluación al año por ser los 6 años una edad promedio en la que erupcionan dichas piezas dentarias. En el ínterin, se hicieron controles cada 3 meses, que permita disminuir las perdidas por seguimiento.

No se evidencian antecedentes en el contexto nacional relacionados a la investigación, pero si en el ámbito internacional; en Colombia, Urrego-Burbano, describe el perfil epidemiológico de la oclusión dental en escolares de Envigado, donde se evaluó la prevalencia de la maloclusión para la dentición permanente (178 escolares) en el año 2010, registrándose que el 49,7 $\%(176 / 354)$ presentaba maloclusión clase I, 43,5\% (154/354) maloclusión clase II y del 6,8 \% (24/354) maloclusión clase III. Con respecto a la dentición decidua, (82 escolares), se encontró un 32,9 \% (27/82)

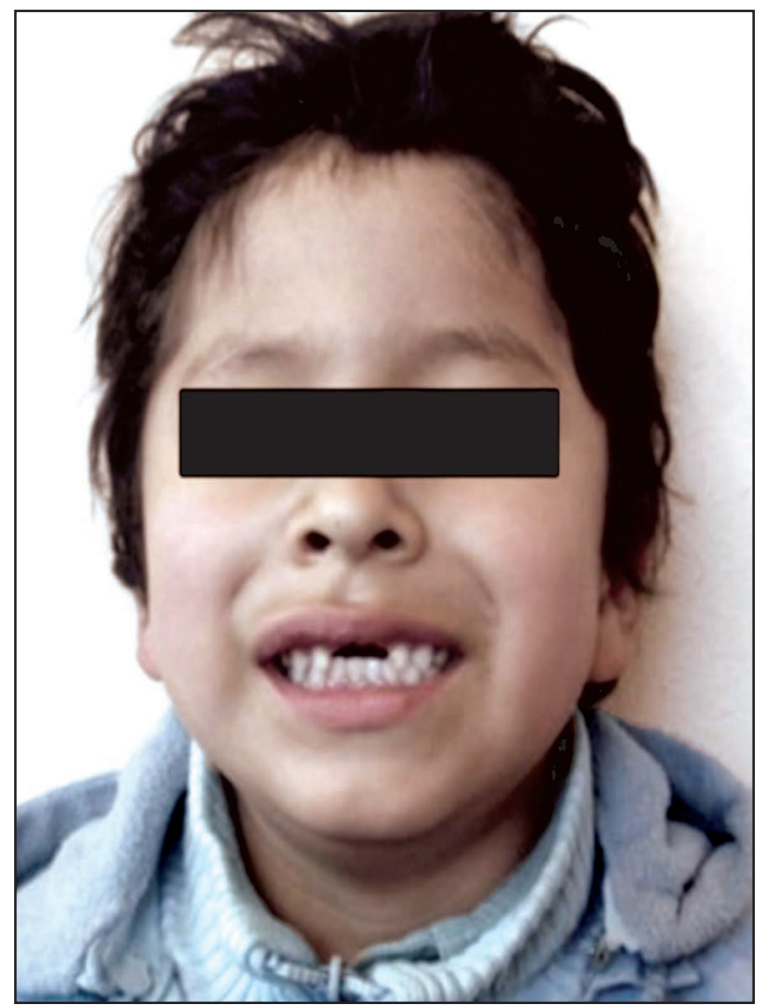

Figura $\mathrm{N}^{\circ}$ 1: Niño seleccionado en el estudio.

con plano terminal recto, un $57,3 \%(47 / 82)$ con escalón mesial y un 9,8 \% (8/82) con escalón distal (1).

En México, Medrano-Luna realizó un estudio epidemiológico para observar la distribución y frecuencia de algunas características de la dentición temporal y valorar el posible riesgo para el desarrollo de algún tipo de maloclusión (2). En éste, examinaron a 193 preescolares de la delegación de Iztapalapa, para valorar el tipo de plano terminal se utilizaron los criterios establecidos por Baume (3) y para la experiencia de caries dental los de OMS.

Los examinadores fueron calibrados (estandarizados) 
de acuerdo a los parámetros establecidos por Cohén, resultando esta calibración casi perfecta. Los resultados fueron: $36,6 \%$ de los preescolares presentaron lesiones cariosas interproximales profundas $y / 0$ pérdida dentaria prematura; no se observó dependencia de estas variables con relación a la edad y sexo. El 81,9\% mostró planos terminales rectos y mesial, de acuerdo a los resultados obtenidos la población mostró bajo riesgo para desarrollar algún tipo de maloclusión. Asimismo, se confirma la naturaleza multifactorial de este proceso, en el cual, algunos otros factores de la dentición temporal pueden tener una influencia mayor en el desarrollo de la oclusión de la dentición permanente.

\section{MATERIAL Y MÉTODOS}

Estudio de nivel descriptivo longitudinal prospectivo. La población estuvo representado por todos los niños de 5 años, que acudieron al servicio de odontología del Hospital Nacional "Daniel Alcides Carrión" de la ciudad de Cerro de Pasco (figura 1, 2). La muestra fue de 40 niños (20 mujeres y 20 varones), escogidos de acuerdo a los criterios de inclusión y exclusión.

Para seleccionar los niños, se hizo un muestreo no probabilístico por criterio, para que la muestra sea más representativa y homogénea fueron escogidos de manera aleatoria.

\section{Criterios de inclusión:}

- Niños de 5 años de edad cumplidos de ambos sexos.

- Niños que presentan los 20 dientes temporales.

- Niños que acudan al Hospital Nacional "Daniel Alcides Carrión".

\section{Criterios de exclusión:}

- Niños con caries proximales.

- Niños en los que no será posible hacer el seguimiento.

- Niños con problemas esqueléticos o congénitos como paladar hendido y labio fisurado.

- Niños que presenten dientes supernumerarios o accesorios en su dentición temporal.

- Niños que presenten malos hábitos orales como chuparse los dedos, masticarse las uñas, lapiceros.

Para que la muestra sea representativa se solicitó el permiso del Hospital en mención para realizar en primer lugar un examen clínico de pre-selección y en segundo lugar para el estudio propiamente dicho con los niños seleccionados, de esos niños pre-seleccionados para el estudio se les comunicó a sus padres mediante el consentimiento informado en el cual se le dio detalles de lo que realizaríamos en el estudio y a la vez lograr el consentimiento para trabajar con sus niños y contar con su apoyo; de estos niños pre-seleccionados se escogerán a los seleccionados propiamente dichos según la información y consentimiento que nos puedan dar los padres. Obtenida la muestra para el estudio se procedió como sigue:

- Los exámenes clínicos solamente fueron objetivos

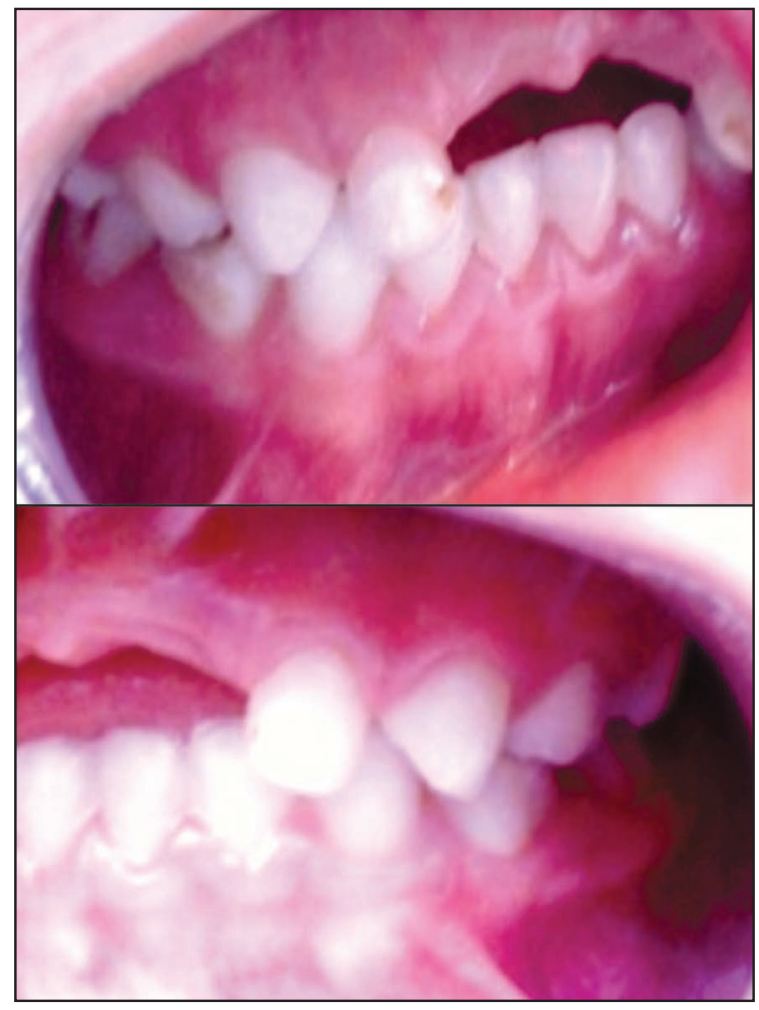

Figura $\mathrm{N}^{\circ}$ 2: Situación a los 5 años.

y no intervencionistas esto quiere decir que solamente observamos características como el tipo de plano Terminal molar, el tipo de arcada de la dentición temporal y consecuentemente las clases de maloclusión (Guía molar) de la dentición permanente.

- Los exámenes clínicos propiamente dichos de la muestra seleccionada fueron en total 5 de los cuales el primero y el último nos darán a conocer de manera determinante lo que queremos conocer en el estudio es por ello que en el instrumento sólo se considera el registro de estos la primera como medición basal y la última como medición post, 
del segundo al cuarto examen solo fue de rutina para evaluar la erupción de la primeras molares permanentes, mantener en buenas condiciones las piezas dentales y como medio para contrarrestar la pérdida de la muestra por seguimiento, además se les condicionó regalándoles un cepillo dental cada vez que vengan a su examen clínico programado.

- Los intervalos para el seguimiento de cada examen clínico desde el primero fueron cada tres meses. Que fue comunicado oportunamente mediante un cronograma a los padres de los niños seleccionados después de la aceptación del consentimiento informado.

\section{RESULTADOS}

En la tabla 1, se muestra la frecuencia del tipo de plano terminal molar de la dentición temporal de cada niño donde se observa que el 52,5\% de niños presentó un tipo de plano terminal molar recto considerado el mayor porcentaje, seguido del plano terminal molar con escalón distal con $30 \%$ y el plano terminal molar con escalón mesial con el menor porcentaje de 17,5 $\%$, llegando a una conclusión que hay una mayor cantidad de niños con plano terminal molar recto y una menor cantidad con plano terminal molar con escalón mesial en la dentición temporal.

Tabla $N^{\circ}$ 1: Frecuencia del tipo de plano terminal molar de la dentición temporal.

\begin{tabular}{lrr}
\hline $\begin{array}{c}\text { Tipo de plano } \\
\text { terminal molar }\end{array}$ & $\mathrm{n}$ & $\%$ \\
\hline Recto & 21 & 52,5 \\
Escalón distal & 12 & 30,0 \\
Escalón mesial & 7 & 17,5 \\
Total & 40 & 100,0 \\
\hline
\end{tabular}

Tabla № 2: Frecuencia del tipo de arcada de la dentición temporal.

\begin{tabular}{lcr}
\hline $\begin{array}{c}\text { Tipo de plano } \\
\text { terminal molar }\end{array}$ & $\mathrm{n}$ & $\%$ \\
\hline Abierta & 26 & 65 \\
Cerrada & 14 & 35 \\
Total & 40 & 100 \\
\hline
\end{tabular}

Tabla № 3: Frecuencia de oclusión de la dentición permanente.

\begin{tabular}{lrr}
\hline $\begin{array}{c}\text { Oclusión de la } \\
\text { dentición permanente }\end{array}$ & $\mathrm{n}$ & $\%$ \\
\hline Normoclusión & 14 & 35 \\
Clase I & 12 & 30 \\
Clase II & 8 & 20 \\
Clase III & 6 & 15 \\
Total & 40 & 100 \\
\hline
\end{tabular}

La tabla 2, muestra la frecuencia del tipo de arcada de la dentición temporal de cada niño donde se observa que el $65 \%$ de niños presentó el tipo de arcada abierta considerado el mayor porcentaje, el tipo de arcada cerrada se consideró con un $35 \%$, llegando a una conclusión que hubo una mayor cantidad de niños con el tipo de arcada abierta en la dentición temporal.

La tabla 3 muestra la distribución de las clases de maloclusión de la dentición permanente. Donde la normoclusión se consigna con el mayor porcentaje un $35 \%$, el tipo maloclusión clase I con el $30 \%$, la clase II se denota con un $20 \%$ y la clase III con el menor porcentaje un $15 \%$. Llegando a una conclusión que hay una mayor cantidad de niños con normoclusión seguida de la maloclusión clase I de la dentición permanente y con una menor cantidad se consigna la maloclusión de clase III.

La tabla 4 muestra la relación del tipo de plano terminal molar de la dentición temporal con la oclusión de la dentición permanente, donde se observa que el tipo de plano terminal molar recto de la dentición temporal se relaciona con mayor frecuencia con la normoclusión y la clase I de maloclusión en un 25 $\%$ y $15 \%$ respectivamente. Mientras que el tipo de plano terminal molar escalón mesial de la dentición temporal representa el menor porcentaje de las diferentes relaciones con las clases de maloclusión de la dentición permanente en un 17,5\%, llegando a la conclusión que el tipo de plano terminal molar recto de la dentición temporal se relaciona frecuentemente con la clase I de maloclusión de la dentición permanente; y el tipo de plano terminal molar escalón mesial se relaciona mayormente con la clase III de maloclusión de la dentición permanente.

La tabla 5 muestra la relación del tipo de arcada de

Tabla № 4: Relación del tipo de plano terminar molar de la dentición temporal y la oclusión de la dentición permanente

\begin{tabular}{|c|c|c|c|c|c|c|c|c|c|c|}
\hline \multirow{3}{*}{$\begin{array}{l}\text { Tipo de plano } \\
\text { terminal molar }\end{array}$} & \multicolumn{10}{|c|}{ Oclusión de la dentición permanente } \\
\hline & \multicolumn{2}{|c|}{ Normoclusión } & \multicolumn{2}{|c|}{ Clase I } & \multicolumn{2}{|c|}{ Clase II } & \multicolumn{2}{|c|}{ Clase III } & \multicolumn{2}{|c|}{ Total } \\
\hline & $\mathrm{n}$ & $\%$ & $\mathrm{n}$ & $\%$ & $n$ & $\%$ & $\mathrm{n}$ & $\%$ & $\mathrm{n}$ & $\%$ \\
\hline Recto & 10 & 25 & 6 & 15 & 3 & 7,5 & 2 & 5 & 21 & 52,5 \\
\hline Escalón distal & 2 & 5 & 4 & 10 & 4 & 10,0 & 2 & 5 & 12 & 30,0 \\
\hline Escalón mesial & 2 & 5 & 2 & 5 & 1 & 2,5 & 2 & 5 & 7 & 17,5 \\
\hline Total & 14 & 35 & 12 & 30 & 8 & 20 & 6 & 15 & 40 & 100,0 \\
\hline
\end{tabular}


Tabla $N^{\circ}$ 5: Relación del tipo de arcada dentición temporal y las clases de maloclusión de la dentición permanente.

\begin{tabular}{|c|c|c|c|c|c|c|c|c|c|c|}
\hline \multirow{3}{*}{$\begin{array}{l}\text { Tipo de } \\
\text { arcada }\end{array}$} & \multicolumn{10}{|c|}{ Oclusión de la dentición permanente } \\
\hline & \multicolumn{2}{|c|}{ Normoclusión } & \multicolumn{2}{|c|}{ Clase I } & \multicolumn{2}{|c|}{ Clase II } & \multicolumn{2}{|c|}{ Clase III } & \multicolumn{2}{|c|}{ Total } \\
\hline & $\mathrm{n}$ & $\%$ & $\mathrm{n}$ & $\%$ & $\mathrm{n}$ & $\%$ & $\mathrm{n}$ & $\%$ & $\mathrm{n}$ & $\%$ \\
\hline Abierta & 10 & 25 & 8 & 20 & 4 & 10 & 4 & 10 & 26 & 65 \\
\hline Cerrada & 4 & 10 & 4 & 10 & 4 & 10 & 2 & 5 & 14 & 35 \\
\hline Total & 14 & 35 & 12 & 30 & 8 & 20 & 6 & 15 & 40 & 100 \\
\hline
\end{tabular}

la dentición temporal con las clases de maloclusión de la dentición permanente. Donde se observó que el tipo de arcada abierta de la dentición temporal se relaciona con mayor frecuencia con la normoclusión y la clase I de maloclusión de la dentición permanente en un $25 \%$ y $20 \%$ respectivamente. Mientras que el tipo de arcada cerrada de la dentición temporal representa el menor porcentaje relacionado con la maloclusión clase III de la dentición permanente con un $5 \%$. Llegando a la conclusión que el tipo de arcada abierta de la dentición temporal se relaciona mayormente con la normoclusión y la clase I de maloclusión de la dentición permanente y el tipo de arcada cerrada de la dentición temporal se relaciona minoritariamente con la clase III de maloclusión de la dentición permanente.

\section{DISCUSIÓN}

La investigación realizada confirma que del total de niños examinados el $52,5 \%$ de niños tuvo un tipo de plano terminal molar recto considerado el mayor porcentaje, seguido del plano terminal molar con escalón mesial con $30 \%$ y el plano terminal molar con escalón distal con el menor porcentaje de 17,5\%.

En México, Medrano-Luna realizó un estudio epidemiológico para observar la distribución y frecuencia de algunas características de la dentición temporal y valorar el posible riesgo para el desarrollo de algún tipo de maloclusión. En éste, examinaron a 193 preescolares de la delegación de Iztapalapa en el año 2007. Los resultados fueron: 36,6 \% de los preescolares presentaron lesiones cariosas interproximales profundas $y / 0$ pérdida dentaria prematura; no se observó dependencia de estas variables con relación a la edad y sexo. El 81,9\% mostró planos terminales rectos y mesial, de acuerdo a los resultados obtenidos la población mostró bajo riesgo para desarrollar algún tipo de maloclusión.

Asimismo, se confirma la naturaleza multifactorial de este proceso, en el cual, algunos otros factores pueden tener una influencia mayor en el desarrollo de la oclusión.

La utilización de la clasificación de Angle para la dentición permanente presenta poca dificultad y ha sido el parámetro para múltiples estudios epidemiológicos (4). En la dentición mixta y en la dentición primaria existen limitaciones para el uso de la misma, derivadas de la falta de establecimiento de la relación del primer molar permanente, el cual puede estar no erupcionado o parcialmente erupcionado. También existen pequeños cambios en la relación molar inherentes al desarrollo de la dentición y al crecimiento de los maxilares. El plano terminal de los segundos molares primarios es de altísimo valor diagnóstico en estas denticiones sobre todo en el sector posterior y el tipo de arcada para el sector anterior. En el presente trabajo el diagnóstico dental de la maloclusión se basa en la relación de la primera molar permanente consecuentemente de la evaluación inicial en el plano terminal molar y tipo de arcada de la dentición temporal. Este diagnóstico se realizó de manera dinámica, tomando en consideración todos los factores correspondientes a la edad y estado del desarrollo de la dentición de cada paciente. Este enfoque sigue siendo altamente reproducible con excelente concordancia una vez realizada la observación.

A partir de los seis años, la dentición temporal va siendo sustituida por la dentición permanente, siendo los primeros molares inferiores los primeros que hacen erupción. La relación anteroposterior entre los dos primeros molares permanentes depende de sus posiciones en los maxilares, la relación sagital entre el maxilar y la mandíbula y los promedios de las dimensiones mesiodistales de las coronas de los molares deciduos, tanto maxilares como mandibulares. Una dentición primaria con diastemas pequeños o prácticamente sin diastemas en la región anterior es una indicación de espacio insuficiente en las arcadas para un recambio sin impedimentos de los incisivos. La distancia intercanina es demasiado pequeña.

El mantenimiento del espacio primate podría darse por la inclinación de las molares superiores. Según Zavaleta de Godoy existe un componente de fuerzas entre los dientes que se mantienen estables en sus posiciones, ejerciendo unos sobre otro una fuerza en sentido contrario (5).

El espaciamiento entre los incisivos primarios es un factor importante que permite que los incisivos permanentes relativamente grandes se acomoden en la arcada. La inclinación vestibular de los incisivos 
permanentes proporciona más espacio, que aumenta el perímetro del arco, y lo mismo sucede con el crecimiento del hueso alveolar, el cual aumenta la amplitud intercanina en la arcada. Debido a que este crecimiento por lo general concluye cuando los incisivos laterales terminan de erupcionar, el apiñamiento no mejora en esa fase del desarrollo; de hecho, puede empeorar en años posteriores por la presión de los dientes posteriores apiñados.

Para ello debemos tener en cuenta cómo es que se establece la dentición permanente. A partir de los 6 años, la dentición temporal va siendo sustituida por la permanente. Antes de que erupcionen los dientes permanentes, el espacio interdental en el área anterosuperior es $2,5 \mathrm{~mm}$ y $1 \mathrm{~mm}$ en el antero-inferior.

Luego de la erupción de los incisivos permanentes el espacio anteriormente citado disminuye. En la maxila hay exceso de espacio mientras que en la mandíbula hay falta de espacio. Después de la erupción de los incisivos laterales hay una falta de espacio de 1,6 mm en la mandíbula, observándose una discrepancia en tamaño, entre incisivos permanentes y deciduos.

El alineamiento de los incisivos inferiores es independiente de la relación de tamaño entre dientes permanentes y deciduos que van a ser reemplazados. Esto es más bien dependiente del aumento del espacio interdental en la dentición decidua y al incremento del perímetro de arco, debido al posicionamiento anterior de los incisivos permanentes.

Al erupcionar los incisivos laterales superiores, estos presionan a los caninos deciduos hacia distal, incrementando así el arco, cerrando el espacio primate y esto a la vez genera una repercusión en el arco superior.

Los incisivos superiores erupcionan hacia labial y presentan un diastema interincisal. El incisivo lateral superior deciduo migra hacia distal (empujado por la presión de la corona de los centrales permanentes sobre su raíz) y ocupa el espacio de primate que ahí existía.

Según Baume, los niños portadores del arco tipo I, con espacios generalizados en la dentición decidua, presentaban un pronóstico más favorable al cambia los incisivos deciduos por los permanentes, es decir no presentarán apiñamiento. Por otro lado los niños portadores del arco tipo II, sin presencia de espacios generalizados, tiene mayor tendencia al apiñamiento en los incisivos permanentes (3).

Las conclusiones de este estudio son:

Hay una mayor cantidad de niños con plano terminal molar recto y una menor cantidad con plano terminal molar con escalón mesial de la dentición temporal.

Hubo una mayor cantidad de niños con el tipo de arcada abierta y en casi la mitad de esta, con el tipo de arcada cerrada de la dentición temporal.

Hay una mayor cantidad de niños con normoclusión seguida de la maloclusión clase I de la dentición permanente y con una menor cantidad se consigna la maloclusión de clase III.

El tipo de arcada abierta se relaciona con mayor frecuencia y por lo tanto con un mayor porcentaje con el tipo de plano terminal molar recto y el mismo tipo de arcada se relaciona con menor frecuencia y porcentaje con el tipo de plano terminal mesial.

El tipo de plano terminal molar recto de la dentición temporal se relaciona frecuentemente con la clase I de maloclusión de la dentición permanente; y el tipo de plano terminal molar escalón mesial se relaciona mayormente con la clase III de maloclusión de la dentición permanente

El tipo de arcada abierta de la dentición temporal se relaciona mayormente con la normoclusión y la clase I de maloclusión de la dentición permanente y el tipo de arcada cerrada de la dentición temporal se relaciona minoritariamente con la clase III de maloclusión de la dentición permanente.

\section{REFERENCIAS BIBLIOGRÁFICAS}

1. Urrego-Burbano P, Jiménez-Arroyave L, LondoñoBolívar M, Zapata- Tamayo M, Botero-Mariaca P. Perfil epidemiológico de la oclusión dental en escolares de Envigado, Colombia. Rev. salud pública. 2011 ; 13(6): 1010-1021.

2. Medrano-Luna J. Distribución y frecuencia de algunas características de la dentición temporal y valorar el posible riesgo para el desarrollo de algún tipo de maloclusión en preescolares de la delegación de Iztapalapa México. Ciencia UANL. 2007; 7: 88-95.

3. Baume L. Physiological tooth migration and its significance for the development of occlusion. I. The biogenic course of the deciduous dentition. J Dent Res. 1950; 29: 123-132.

4. Angle EH. Classification of malocclusion. Dental Cosmos. 1899; 41(18): 248-263.

5. Zabaleta de Godoy V, Chelotti A, Matson E. Contribucao oa estudo das inclinacoes das faces oclusais dos primeros molares deciduos inferiores em relacao as dos segundos molares decíduos inferiores. Rev Fac Odont. 1986; 24(2): 57-61. 